Published in final edited form as:

Cancer Causes Control. 2017 March ; 28(3): 235-240. doi:10.1007/s10552-017-0860-8.

\title{
Prostate cancer screening decision-making in three states: 2013 behavioral risk factor surveillance system analysis
}

\author{
Jun $\mathrm{Li}^{1}{ }^{1}$, Ingrid J. Hall ${ }^{1}$, and Guixiang Zhao ${ }^{2}$ \\ 1Division of Cancer Prevention and Control, National Center for Chronic Disease Prevention and \\ Health Promotion, CDC, 4770 Buford Hwy, Mail Stop F-76, Atlanta, GA 30341, Georgia \\ 2Division of Population Health, National Center for Chronic Disease Prevention and Health \\ Promotion, CDC, Atlanta, Georgia
}

\begin{abstract}
Introduction-Given the discordant prostate cancer screening recommendations in the United States, shared decision-making (SDM) has become increasingly important. The objectives of this study were to determine who made the final decision to obtain prostate-specific antigen (PSA)based screening and identify factors associated with the screening decision made by both patients and their health care providers.
\end{abstract}

Methods-Using the 2013 Behavioral Risk Factor Surveillance System data from Delaware, Hawaii, and Massachusetts, we calculated weighted percentages of SDM. Associations between the SDM and sociodemographic, lifestyle, access to care, and PSA testing-related factors were assessed using multivariate logistic regression.

Results-There were 2,248 men aged 40 years or older who ever had a PSA-based screening in these three states. Only $36 \%$ of them made their prostate cancer screening decision jointly with their health care provider. Multivariate analyses showed that men who were married/living together or had a college degree and above were more likely to report having SDM than men who were never married or had less than high school education $(P=0.02$ and 0.002$)$. Moreover, men whose most recent PSA test occurred within the past year were more likely to report SDM than men who had the test done more than 2 years ago $(P=0.02)$.

Conclusions-The majority of screening decisions were made by the patient or health care provider alone in these three states, not jointly, as recommended. Our study points to the need to promote SDM among patients and their health care providers before PSA testing.

\section{Keywords}

Shared decision-making; Prostate cancer screening; Prostate-specific antigen test

Correspondence to: Jun Li.

Compliance with ethical standards

Disclaimer The findings and conclusions in this report are those of the authors and do not necessarily represent the official position of CDC.

Conflict of interest No financial disclosures were reported by the authors of this paper. 


\section{Introduction}

Due to conflicting evidence for the effectiveness of the prostate-specific antigen (PSA) test in reducing mortality and the known potential harms, in 2012, the United States Preventive Services Task Force (USPSTF) recommended against routine PSA-based prostate cancer screening for men of all ages. In addition, the USPSTF recommends that physicians help patients who clearly express an interest in PSA testing make an informed decision before ordering a test $[1,2]$. Currently, prostate cancer screening recommendations vary markedly among major medical societies in the United States [2-5]. However, informed or shared decision-making, a communication process on the benefits, harms, and scientific uncertainties of PSA testing conducted between health care providers and patients to help patients make value-concordant decisions about screening, has consistently been recommended [6].

National evidence indicated that only one-third of men reported ever having past discussions about advantages, disadvantages, and the scientific uncertainties of PSA-based screening with their health care providers [7]. Imbalanced discussions, which emphasize the advantage of the test more frequently and adequately than its disadvantages, have been well documented in the literature [7-10]. Information about who made the final decision to have a PSA test is scarce. In 2013, the Centers for Disease Control and Prevention collected data about prostate cancer screening decision-making through the Behavioral Risk Factor Surveillance System (BRFSS). The objectives of this study were to determine who made the final decision to obtain a PSA test and identify factors associated with shared decisionmaking between patients and their health care providers.

\section{Methods}

This study used data from the 2013 BRFSS, a state-based telephone survey of health risk behaviors, preventive health practices, and health care access among the noninstitutionalized US civilian population aged 18 years or older in the 50 states and the District of Colombia (http://www.cdc.gov/BRFSS/). Delaware, Hawaii, and Massachusetts were the only states that collected information about prostate cancer screening decision-making among men aged 40 years or older who ever had a PSA test and yielded response rates of 40.1, 40.2, and $39.9 \%$, respectively.

As shown in Appendix I, the primary outcome of this analysis was defined as a shared decision to screen determined by both men and their health care providers before ordering a PSA test (hereafter SDM). Briefly, SDM was classified as "yes" only for those responding C to Question 1 and A to question 2. Associations of the SDM (yes vs no) with sociodemographics (age, race/ethnicity, education, employment, and marital status), lifestyles (smoking and exercise), and access to care factors (have health insurance and regular health care provider) along with ever having discussed PSA testing (yes vs no) and time of receiving most recent test were examined (within a year, 1 year but less than 2 years, or 2 years and more). Survey questions about if ever been discussed the advantages and disadvantages of the PSA test and about the time, since the last PSA test were shown in Appendix II. We identified SDM for prostate cancer screening (not for cancer diagnostic or 
surveillance purposes) based on responding A and C to survey question 4 in the Appendix II: "What was the Main reason you had this PSA test—was it...?"

We analyzed three states combined data using SAS-callable SUDAAN, version 9.3, to account for the multistage and disproportionate stratified sampling design. We conducted bivariate analysis to estimate unadjusted percentages of SDM with 95\% confidence intervals (CIs) by the aforementioned characteristics. The main effect of the unadjusted percentage was assessed by the Chi-square test. Adjusted percentages with 95\% CIs for SDM were calculated using multivariate logistic regression controlling for all covariables. Predicted margins for each subgroup were calculated from the logistic regression model as the average of the predicted probabilities of SDM, assuming that all survey participants were in that subgroup.

\section{Results}

As shown in Table 1, there were 2,248 men aged 40 years or older who ever had a PSA test for cancer screening in these three states in 2013. Among them, only 36\% of men made their prostate cancer screening decision jointly with their health care providers; $25 \%$ of men made the decision on their own, and $37 \%$ of men had the decision made by their health care provider.

Bivariate analysis showed that shared decision-making for prostate cancer screening was associated with men's educational attainment and marital status (Table 2). Multivariate analyses revealed that men who were married/living together or had a college degree and above were more likely to report having SDM on PSA testing than men who were never married or had less than high school education ( $P=0.02$ and 0.002). SDM was reported more frequently for men who had no health insurance $(P<0.001)$. In addition, men whose most recent PSA test occurred within the past year were more likely to report SDM than men who had the test done more than 2 years ago $(P=0.02)$.

\section{Discussion}

On the basis of the 2013 BRFSS data, our study suggests that SDM of prostate cancer screening remains substantially underused-only $36 \%$ among men who got screened. However, we found that men who had PSA testing within the past year were more likely to report shared decision-making than men who had the test more than 2 years ago. This might reflect an early improvement in conducting SDM in these three states. Recently published prostate cancer screening recommendations emphasizing the importance of SDM may partially account for the changes [2-5]. A national internet survey among patients who had prostate cancer screening discussions found the majority of final screening decisions which were made by either the health care provider or patient alone in 2011 - only $34 \%$ of the decisions were made by patients and their health care provider together [9].

Given the uncertain effectiveness of PSA testing for reducing prostate cancer mortality, and the potential harms associated with diagnosing and treating prostate cancer cases that will not progress or progress slowly, SDM plays an important role in helping patients make decisions that are most consistent with their values and preferences. However, national 
surveillance data showed that about $65 \%$ of all survey participants, who were aged 50-74 years, had no screening discussion with their health care providers in 2010 [7]. Among men who had prostate screening discussions, most discussions addressed the advantages of PSA testing; fewer addressed the disadvantages of the test $[8,9]$. According to the 2010 National Health Interview Survey data, only $27 \%$ of men aged 70 years or older who underwent PSA testing reported having discussions about both advantages and disadvantages of the test [10]. SDM before PSA testing has been underused between patients and their health care providers. Studies have indicated that decision-aids to help patients make informed decisions have been shown to improve their prostate cancer knowledge and reduce decision conflicts $[11,12]$. Other potential strategies to promote SDM include changing outpatient clinic visit reimbursement, providing pre-visit decision support interventions, and physician education on value-concordant decision-making [13].

Our study suggested that men who had higher education, were married/living together, or had no health insurance were more likely to report having SDM before screening. Positive associations of higher education and married/living together with PSA testing have been reported, [10, 14]; however, their associations with SDM before PSA testing are understudied [7, 15]. Using the 2010 NHIS data, Han et al. reported that higher education and having private/military insurance were positively associated with discussion of uncertainty of PSA testing, but not discussion of advantages and disadvantages of the test [7]. Several factors may partially explain the differences between our findings and theirs: (1) our study population only included men who undertook a PSA test, instead of all men regardless of having the test; (2) we classified the health insurance variable as "Yes" or "No" instead of specifying insurance type; (3) we controlled marital status in the model, but they did not. More studies are needed to better understand the predictors for SDM, including education levels, and marital and health insurance status.

This study is subject to several limitations. First, the BRFSS data were self-reported and, thus, subject to recall bias. Second, low response rates increase the potential for selection bias; however, the calculations of weights, which were adjusted for differences in probability of selection and nonresponse, might have partially corrected the bias. Third, PSA testing may be underreported in the survey, because the test may have been conducted without the patient being told by his physician [16]. Fourth, this study has limited generalizability, because data were from three states. Finally, respondents might comprehend survey questions about prostate cancer screening decision-making differently [17]; thus, measurement errors could not be ruled out.

\section{Conclusions}

Although our data suggest that more SDM occurred in recent years, only one-third of men reported having SDM on prostate cancer screening with their health care provider in Delaware, Hawaii, and Massachusetts in 2013. Our study points to the need to promote SDM among patients and their health care providers before ordering a PSA test. 


\section{Acknowledgments}

The findings and conclusions in this report are those of the authors and do not necessarily represent the official position of CDC.

\section{References}

1. Force USPSTTalking With Your Patients About Screening for Prostate Cancer2012

2. Moyer VA. Force USPST. Screening for prostate cancer: U.S. Preventive Services Task Force recommendation statement. Ann Intern Med. 2012; 157:120-134. [PubMed: 22801674]

3. Carter HB, Albertsen PC, Barry MJ, et al. Early Detection of Prostate Cancer: AUA Guideline. J Urol. 2013; 190:419-426. [PubMed: 23659877]

4. Qaseem A, Barry MJ, Denberg TD, Owens DK, Shekelle P. Clinical Guidelines Committee of the American College of P. Screening for prostate cancer: a guidance statement from the Clinical Guidelines Committee of the American College of Physicians. Annals of internal medicine. 2013; 158:761-769. [PubMed: 23567643]

5. Smith RA, Manassaram-Baptiste D, Brooks D, et al. Cancer screening in the United States, 2014: a review of current American Cancer Society guidelines and current issues in cancer screening. CA Cancer J Clin. 2014; 64:30-51. [PubMed: 24408568]

6. Knight SJ. Decision making and prostate cancer screening. Urol Clin North Am. 2014; 41:257-266. [PubMed: 24725488]

7. Han PK, Kobrin S, Breen N, et al. National evidence on the use of shared decision making in prostate-specific antigen screening. Ann Fam Med. 2013; 11:306-314. [PubMed: 23835816]

8. Hoffman RM, Couper MP, Zikmund-Fisher BJ, et al. Prostate cancer screening decisions: results from the National Survey of Medical Decisions (DECISIONS study). Arch Intern Med. 2009; 169:1611-1618. [PubMed: 19786681]

9. Hoffman RM, Elmore JG, Fairfield KM, Gerstein BS, Levin CA, Pignone MP. Lack of shared decision making in cancer screening discussions: results from a national survey. Am J Prev Med. 2014; 47:251-259. [PubMed: 24923862]

10. Li J, Berkowitz Z, Richards TB, Richardson LC. Shared decision making in prostate-specific antigen testing with men older than 70 years. Journal of the American Board of Family Medicine : JABFM. 2013; 26:401-408. [PubMed: 23833155]

11. Taylor KL, Williams RM, Davis K, et al. Decision making in prostate cancer screening using decision aids vs usual care: a randomized clinical trial. JAMA Internal Med. 2013; 173:17041712. [PubMed: 23896732]

12. Volk RJ, Hawley ST, Kneuper S, et al. Trials of decision aids for prostate cancer screening: a systematic review. Am J Prev Med. 2007; 33:428-434. [PubMed: 17950409]

13. Wilkes M, Srinivasan M, Cole G, Tardif R, Richardson LC, Plescia M. Discussing uncertainty and risk in primary care: recommendations of a multi-disciplinary panel regarding communication around prostate cancer screening. J Gen Internal Med. 2013; 28:1410-1419. [PubMed: 23649782]

14. Li J, Zhao G, Hall IJ. Pre-screening discussions and prostate-specific antigen testing for prostate cancer screening. Am J Prev Med. 2015; 49:259-263. [PubMed: 25997905]

15. McFall SL. US men discussing prostate-specific antigen tests with a physician. Ann Fam Med. 2006; 4:433-436. [PubMed: 17003144]

16. Chan EC, Vernon SW, Ahn C, Greisinger A. Do men know that they have had a prostate-specific antigen test? Accuracy of self-reports of testing at 2 sites. Am J Public Health. 2004; 94:13361338. [PubMed: 15284039]

17. Wunderlich T, Cooper G, Divine G, et al. Inconsistencies in patient perceptions and observer ratings of shared decision making: the case of colorectal cancer screening. Patient Educ Couns. 2010; 80:358-363. [PubMed: 20667678] 


\section{Appendix 1}

Prostate Cancer Screening Decision-Making Module.

1. Which one of the following best describes the decision to have the PSA test done?

A. You made the decision alone. [Go to next module].

B. Your doctor, nurse, or health care provider made the decision alone. [Go to next module].

C. You and one or more other persons made the decision together.

D. You do not remember how the decision was made. [Go to next module].

2. Who made the decision with you? (Mark all that apply).
A. Doctor, nurse, or health care provider.
B. Spouse or significant other.
C. Other family member.
D. Friend or nonrelative.

\section{Appendix 2}

Prostate Cancer Screening Module:

1. A Prostate-Specific Antigen test, also called a PSA test, is a blood test used to check men for prostate cancer. Has a doctor, nurse, or other health professional EVER talked with you about the advantages of the PSA test?
A. Yes.
B. No.

2. Has a doctor, nurse, or other health professional EVER talked with you about the disadvantages of the PSA test?
A. Yes.
B. No.

3. How long has it been since you had your last PSA test?
A. Within the past year (anytime less than 12 months ago).
B. Within the past 2 years (1 year but less than 2 years).
C. Within the past 3 years (2 years but less than 3 years).
D. Within the past 5 years (3 years but less than 5 years).
E. 5 or more years ago.

4. What was the main reason you had this PSA test - was it...? 

A. Part of routine exam.
B. Because of a prostate problem.
C. Because of a family history of prostate cancer.
D. Because you were told you had prostate cancer.
E. Some other reason. 


\section{Table 1}

Participant characteristics and weighted percentages for men who ever had a PSA test for cancer screening in Delaware, Hawaii, and Massachusetts, BRFSS 2013

\begin{tabular}{|c|c|}
\hline & Weighted $\%(n=2,248$; weighted $N=533,942)$ \\
\hline \multicolumn{2}{|l|}{ Age } \\
\hline $40-54$ years & 25.8 \\
\hline $55-69$ years & 51.9 \\
\hline $70+$ years & 22.3 \\
\hline \multicolumn{2}{|l|}{ Race/ethnicity } \\
\hline Non-Hispanic white & 78.8 \\
\hline Non-Hispanic black & 6.1 \\
\hline Hispanic & 5.2 \\
\hline Non-Hispanic other & 9.9 \\
\hline \multicolumn{2}{|l|}{ Education } \\
\hline College graduate & 45.4 \\
\hline Some college & 23.8 \\
\hline High school graduate & 23.6 \\
\hline Less than high school & 7.2 \\
\hline \multicolumn{2}{|l|}{ Employment } \\
\hline Employed & 57.0 \\
\hline Unemployed & 4.4 \\
\hline Retired & 32.8 \\
\hline Others & 5.8 \\
\hline \multicolumn{2}{|l|}{ Marital status } \\
\hline Married or living together & 77.8 \\
\hline Divorced, separated, and widowed & 14.3 \\
\hline Never married & 7.9 \\
\hline \multicolumn{2}{|l|}{ Smoking status } \\
\hline Current & 9.3 \\
\hline Former or never & 90.8 \\
\hline \multicolumn{2}{|l|}{ Regular exercise } \\
\hline Yes & 79.2 \\
\hline No & 20.8 \\
\hline \multicolumn{2}{|l|}{ Have health insurance } \\
\hline Yes & 98.0 \\
\hline No & 2.0 \\
\hline \multicolumn{2}{|l|}{ Have a primary doctor } \\
\hline Yes & 97.2 \\
\hline No & 2.8 \\
\hline \multicolumn{2}{|c|}{ Ever discussed advantages and disadvantages } \\
\hline Yes & 42.4 \\
\hline No & 57.6 \\
\hline
\end{tabular}




\begin{tabular}{ll}
\hline & Weighted \% $(\boldsymbol{n}=\mathbf{2 , 2 4 8}$; weighted $N=\mathbf{5 3 3 , 9 4 2})$ \\
\hline When had a PSA test & 63.4 \\
Within a year & 16.2 \\
1 year but less than 2 years & 20.4 \\
2 years and more & 25.0 \\
Who made the decisions to obtain PSA testing \\
Patient only & 37.0 \\
Health care provider only & 36.4 \\
Patient and health care provider & 1.6 \\
Patient and others & \\
\hline
\end{tabular}

$P S A$ prostate-specific antigen

Percentages may not add to $100 \%$ because of rounding

${ }^{a}$ Others include spouse or significant other, other family member, and friend or nonrelative 


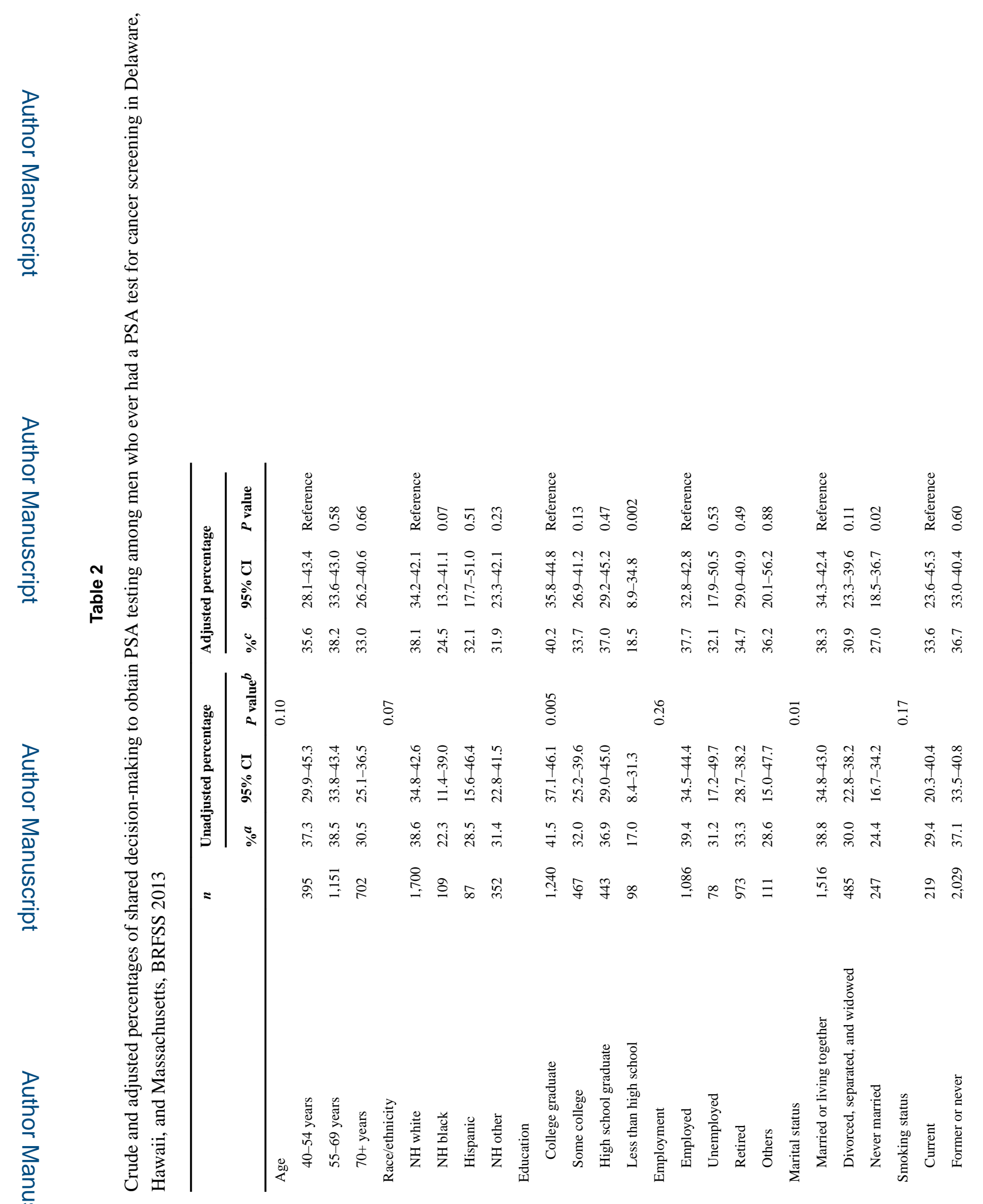




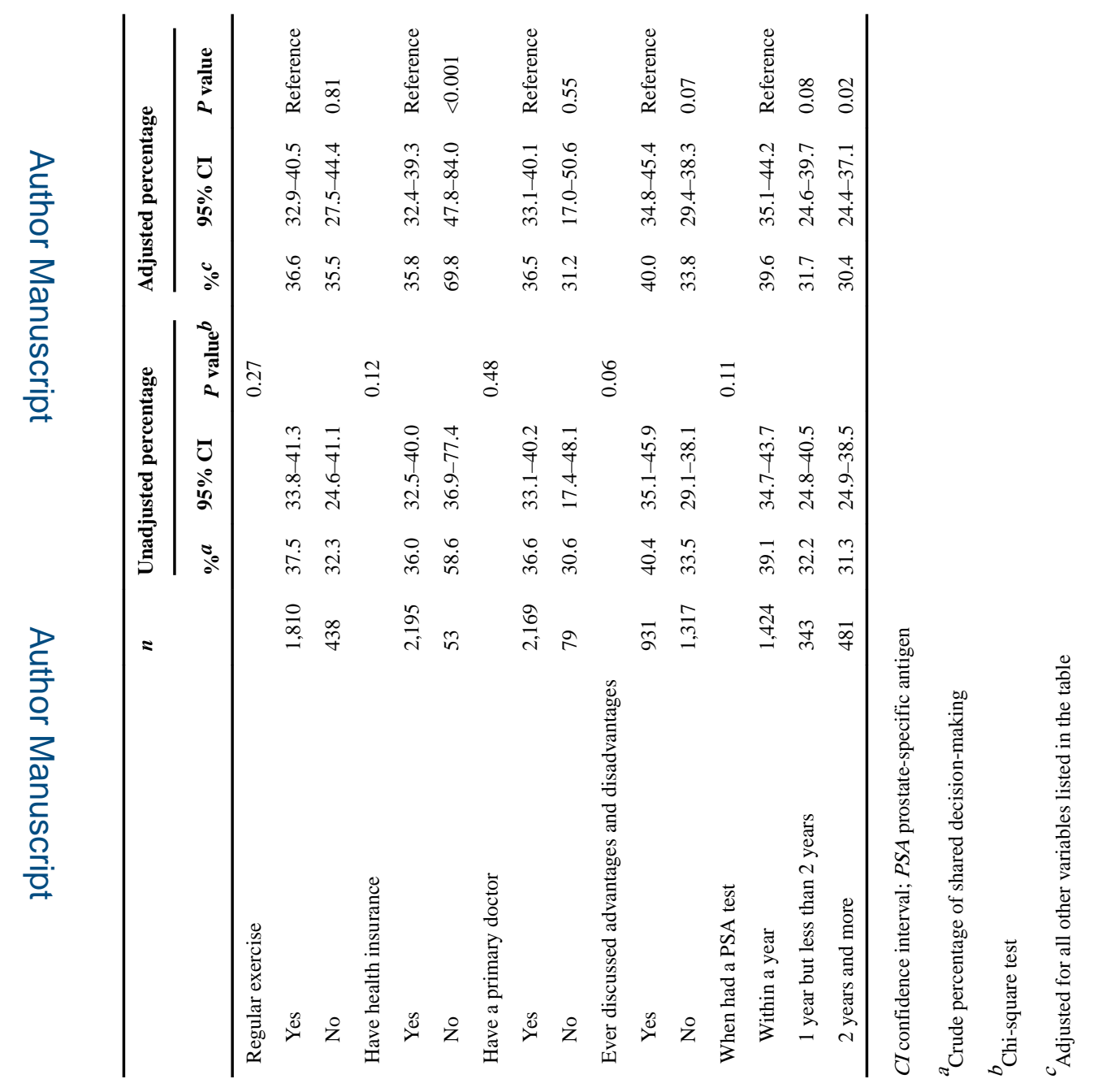

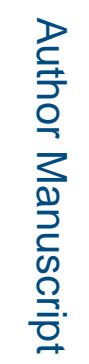

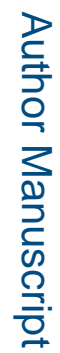

Cancer Causes Control. Author manuscript; available in PMC 2018 July 30. 